\title{
Multi-material Heatsink Design using Level-set Topology Optimization
}

\author{
Mani sekaran Santhanakrishnan, Tim Tilford, Member, IEEE and Chris Bailey, Senior Member, IEEE
}

\begin{abstract}
In this article we apply a Level-set topological optimization algorithm to the design of multi-material heat sinks suitable for electronics thermal management. This approach is intended to exploit the potential of metal powder additive manufacturing technologies which enable fabrication of complex designs. The article details the state-of-the-art in topological optimization before defining a numerical framework for optimization of two-material and three-material based heatsink designs. The modelling framework is then applied to design a pure copper and a copper-aluminum heatsink for a simplified electronics cooling scenario and the performance of these designs are compared. The benefits and drawbacks of the implemented approach are discussed along with enhancements that could be integrated within the framework. A benchmarking study is also detailed which compares the performance of topologically optimized heat sink against a conventional pin-fin heat sink. This is the first time that topological optimization methods have been assessed for multi-material heat sink design where both conduction and convection are included in the analysis. Hence, the reported work is novel in its application of a state-of-the-art Levelset topology optimization algorithm to design multi-material structures subject to forced convective cooling. This paper is intended to demonstrate the applicability of topological optimization to the design of multi-material heatsinks fabricated using additive manufacturing processes and succeeds in this objective. The paper also discusses challenges, which need to be addressed in order to progress this modelling as a design approach for practical engineering situations. The presented methodology is able to design thermal management structures from a combination of aluminum and copper that perform similarly to pure copper but utilizing less expensive materials resulting in a cost benefit for electronics manufacturers.
\end{abstract}

Index Terms-Topological Optimization, Microelectronics, Thermal Management, Engineering Design, Level-set Method

\section{INTRODUCTION}

$\mathrm{T}$ opological optimization algorithms tend to develop designs which are complex and organic in nature and are often difficult to manufacture using traditional methods. These manufacturing challenges can be readily overcome using new additive manufacturing approaches and, as such, topological optimization and additive manufacturing can be considered to be highly synergistic. The ability to additively manufacture parts from a combination of metal powders enables spatial variation of material properties which may enhance either the

This article was submitted for review on the $22^{\text {nd }}$ of August, 2018. M. Santhanakrishnan, T. Tilford and C. Bailey are with the Department of Mathematical Sciences, University of Greenwich, London SE10 9LS, UK. M. Santhanakrishnan is currently with Cranfield University, MK43 OAL, UK performance of the component or, more pragmatically, the price-performance trade-off of the component. This study aims to apply topological optimization to form a heatsink design that combines high cost materials such as copper with low(er) cost materials such as aluminum in less critical areas.

Topological optimization (TO) techniques can be utilized to determine the optimal distribution of one or more materials within the given design space subject to a prescribed set of constraints [1]. The field of topological optimization was pioneered by Bendsøe and Sigmund [2] who focused on applications in structural design. The algorithms underpinning this work, and much of the subsequent research, are based on the Density Method optimization approach coupled with the Method of Moving Asymptotes [3] optimizer. This approach, without regularization, leads to areas of the design domain that are partially fluid and partially solid, leading to inaccuracy in material boundary definition. The Level-set method (LSM) is an alternative approach for topology optimization which utilizes an auxiliary function, called the level-set function to represent a surface. This approach has been applied for topological optimization of structural problems $[4,5]$ since 2003. The approach is slightly more complex than the Density Method but provides sharper capture of interfaces and precludes inter-material (grey) regions through frequent reinitializations of level-sets.

Topological optimization of fluid flow problems was initially based on the Density Method approach presented in the work of Borevall and Petersson [6] and Olesen et al. [7]. Subsequent use of the level-set approach for optimization of fluid flow problems was led by Challis and Guest [8] and extended by Zhou and $\mathrm{Li}$ [9] and integrated with the extended finite element method (xFEM) analysis by Kreissl and Maute [10]. While density and level-set methods are the most popular approaches, research has taken place on various other topology optimization methods, including topology derivative method, phase field approaches, and evolutionary structural optimization method.

Multi- material topology optimization based on the density method has been applied to structural problems by many researchers, including Sigmund and Torquato [11]. Wang and Wang [12] presented a level set (LS) based multi-material method for structural optimization and recently Y. Wang et al. [13] proposed a simple and effective multi-material Level-set formulation. Allaire et al. [14] gave a more rigorous shape

Contact email: M.Santhanakrishnan@cranfield.ac.uk Funding for this study has been provided through the University of Greenwich Vice Chancellor's scholarship scheme. 
derivative for the multi-material topology optimization problems. Generally in multi-material problems the material interface between two solids is assumed to be perfectly bonded, but this need not be the case in practice. Michailidis [15] gives a description of different methods for modelling the material interface with relevant numerical examples.

In addition to the density and the level set methods, a number of other methods have also been applied to multimaterial topology optimization. These include the peak function method of Yin and Ananthasuresh [16], the bi-value coding parameterization scheme of Gao et al. [17] and the shape function approach proposed by Bruyneel [18]. Phase-field approaches based on the Cahn-Hilliard equation are adopted by Tavakoli and Mohseni [19] and by Zhou and Wang [20]. The primary drawback of these approaches is their slow convergence rate with thousands of iterations typically required to achieve a good level of convergence.

Topological optimization of a single material heatsink design has been performed by Dede [21] who optimized the liquid cooling channels for a rectangular domain with a volumetric heat source without interpolating the thermal properties of solid and fluid. Yoon [22] carried out the design of a heat dissipating structure subjected to forced convection with the interpolation of material properties. Dede et al. [23] designed 3D air cooled heat sinks considering conduction and simplified side surface convection. Other notable works on single material heat sink design also include the works of Alexanderson [24] using the density method and by Yaji [25] and Coffin [26] using the levelset method. An alternate topological design approach for heatsink optimization has been presented by Bornoff et al. The method is based on Bejan's constructal theory [27], which explains the underlying principle behind all naturally existing designs or configurations. Bornoff utilizes the approach as both an additive design method [28] and as a subtractive design method [29] for heatsink designs. In the former study, material is sequentially added at the maximum temperature region and in the later from a baseline heat sink, material is sequentially removed where the bottle neck number is lowest. Lasance and Poppe [30] provides an industry point of overview about heat sinks and discusses about various methods (empirical, CFD and testing) to evaluate the heat sink performance and their pros and cons.

Zhuang et al. [31] presented a method for the multi-material optimization of heat conduction problems based on 'color-level set' approach and with the use of the adjoint method for evaluation of shape sensitivity. Additionally, Long et al. [32] presented an efficient quadratic approximation based optimizer for the multi-material topology optimization of transient heat conduction problems. A consolidated review of heat transfer related topology optimization research is presented by Dbouk [33].

The current state-of-the art for multi-material heatsink design solely focuses on conductive heat transfer with no fluid flow. This article extends beyond this by considering combined convective and conductive heat transfer as would be found in typical electronics thermal management problems. The
TABLE I

NOMENCLATURE

\begin{tabular}{lll}
\hline \hline Symbol & \multicolumn{1}{c}{ Quantity } & Unit \\
\hline$\psi$ & Signed Distance Function & - \\
$\rho$ & Density & $\mathrm{Kg} \cdot \mathrm{M}^{-3}$ \\
$u$ & Fluid flow velocity & $\mathrm{M} \cdot \mathrm{s}^{-1}$ \\
$\mu$ & Fluid dynamic viscosity & $\mathrm{Pa} \cdot \mathrm{s}$ \\
$\alpha$ & Brinkman porosity term & - \\
$\mathrm{C}_{\mathrm{p}}$ & Specific heat capacity & $\mathrm{J} \cdot \mathrm{Kg}^{-1} \cdot \mathrm{K}^{-1}$ \\
$\mathrm{k}$ & Thermal conductivity & $\mathrm{W} \cdot \mathrm{M}^{-1} \cdot \mathrm{K}^{-1}$ \\
$\mathrm{~T}$ & Temperature & $\mathrm{K}$ \\
$Q$ & Heat energy flux & $\mathrm{W} \cdot \mathrm{M}^{-2}$ \\
$\mathrm{H}$ & Heaviside function & - \\
$\delta$ & Heaviside derivative & - \\
$\mathrm{h}$ & Heaviside function bandwidth & $\mathrm{M}$ \\
$\mathrm{V}$ & Volume constraint & $\mathrm{M}^{3}$ \\
$\mathrm{~F}$ & Objective function & $\mathrm{WKM} \mathrm{M}^{-3}$ \\
$\lambda$ & Lagrangian multiplier & - \\
$\Lambda$ & Volume penalty factor & - \\
$\beta$ & Volume penalty update factor & - \\
$\mathrm{F}$ & Shape sensitivity & - \\
$\mathrm{L}$ & Domain Length & $\mathrm{M}$ \\
$\mathrm{W}$ & Domain width & $\mathrm{M}$ \\
$\mathrm{H}$ & Domain height & $\mathrm{M}$ \\
$\Omega$ & Design domain & - \\
$\mathrm{Re}$ & Reynolds number & - \\
Subscripts & & \\
1 & Material 1 or Solid1 (Copper) & \\
2 & Material 2 or Solid2 (Aluminum) & \\
$\mathrm{n}$ & Normal component & \\
$\mathrm{s}$ & Solid & \\
$\mathrm{f}$ & Fluid & \\
\hline
\end{tabular}

numerical approach adopted in this work is an extension of the multi-material level set model recently proposed by Y. Wang [17]. The model is applied to the design of forced convection cooled multi-material heat sinks for a number of combinations of Copper and Aluminum. The numerical model is formulated using Matlab [34] to manage the optimization process in combination with the COMSOL Multiphysics package [35] which is used for analysis of thermo-physical aspects of the problem. In this paper, section II describes the two material level set formulation, section III describes the three material formulation, and section IV outlines the computational details. Results of multi-material heat sink design study and its discussion are given in section $\mathrm{V}$ along with the results of a benchmarking study. The conclusions are given in section VI.

\section{TWO-MATERIAL LEVEL SET TOPOLOGY OPTIMIZATION MODEL}

The aim of the optimization methodology is to determine the arrangement of material within a defined design space that best fits a prescribed objective. In this work, a numerical domain is defined within the COMSOL package and subsequently discretized into a large number of finite elements. This domain covers the entire thermo-fluid analysis volume. Inside this domain, a 'design domain' where heat sink shape is to be developed using level set topology optimization, is defined.

Level set functions are used to represent the interface boundary between any two different materials and were initially used to study crack propagation in solids and multiphase flows [36]. Mathematically, a level-set of a differentiable function ' $\mathrm{f}$ ' corresponding to a real value ' $c$ ' is the set of points which 
satisfies the condition $\mathrm{f}=\mathrm{c}$. For example, for a quadratic function in 2D, level-set is a plane curve (a conic section) and in $3 \mathrm{D}$ it is a level surface. In this two material topology optimization model, two level-set functions (LSF) are used to model the two different solids and a fluid. Signed Distance Functions (SDF) are used as level set functions in this study and as per its name, this function value at any point, is equal to the Euclidean distance of that point from a specified boundary. The first LSF $(\psi 1)$ is used to differentiate between solid and fluid, with a positive value considered to represent the solid and negative value considered to represent the fluid. A second $\operatorname{LSF}(\psi 2)$ is used to differentiate between the two solids. The correlation between the LSFs and different materials is illustrated in Figure 1.

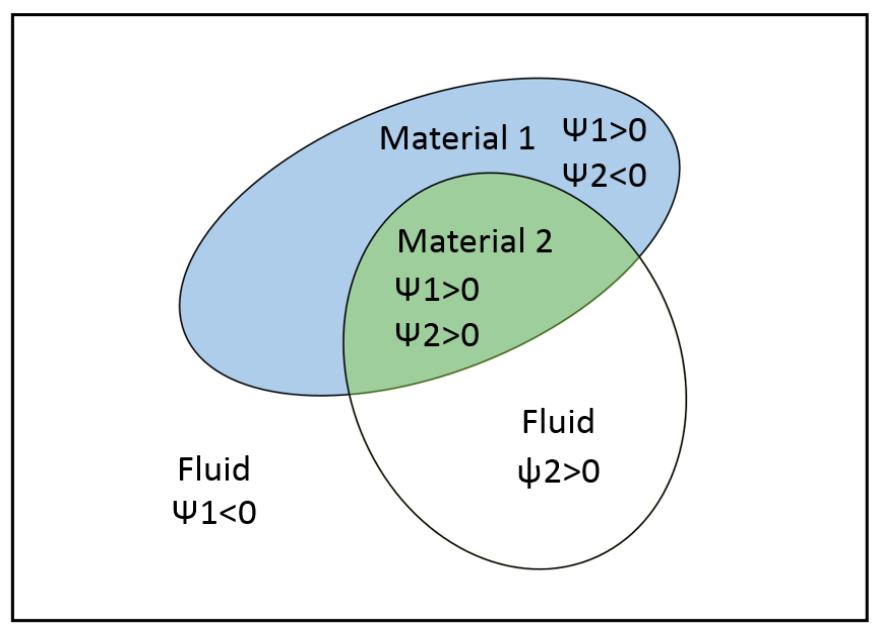

Fig. 1. Design domain and level set function definitions

Since optimization is taking place only within the design domain, level set functions are initialized only within the design domain. The governing equations for the thermo-fluid problem are as follows:

\section{Momentum Conservation}

$$
\begin{gathered}
\rho_{\gamma}(u \cdot \nabla u)=-\nabla p+\nabla \cdot\left\{\mu\left\{\nabla u+(\nabla u)^{T}\right\}\right\}-\alpha u \\
\rho_{\gamma}(\nabla \cdot u)=0
\end{gathered}
$$

TABLE II

\begin{tabular}{|c|c|c|}
\hline Property & Notation & Expression \\
\hline $\begin{array}{l}\text { Thermal } \\
\text { conductivity }\end{array}$ & $\mathbf{K}_{\gamma}$ & $\mathrm{H}_{1} *\left(\mathrm{H}_{2} * \mathrm{k}_{\mathrm{s} 2}+\left(1-\mathrm{H}_{2}\right) * \mathrm{k}_{\mathrm{s} 1}\right)+\mathrm{k}_{\mathrm{f}} *\left(1-\mathrm{H}_{1}\right)$ \\
\hline $\begin{array}{l}\text { Specific heat } \\
\text { capacity }\end{array}$ & $\mathrm{Cp} \gamma$ & $\mathrm{H}_{1} *\left(\mathrm{H}_{2} * \mathrm{cp}_{\mathrm{s} 2}+\left(1-\mathrm{H}_{2}\right) * \mathrm{cp}_{\mathrm{s} 1}\right)+\mathrm{c}_{\mathrm{pf}} *\left(1-\mathrm{H}_{1}\right)$ \\
\hline Density & $\rho_{\gamma}$ & $\mathrm{H}_{1} *\left(\mathrm{H}_{2} * \rho_{\mathrm{s} 2}+\left(1-\mathrm{H}_{2}\right)^{*} \rho_{\mathrm{s} 1}\right)+\rho_{\mathrm{f}}^{*} *\left(1-\mathrm{H}_{1}\right)$ \\
\hline $\begin{array}{l}\text { Impermeabilit } \\
\text { y factor }\end{array}$ & $\alpha$ & $\left(\alpha_{\max }-\alpha_{\min }\right) * H_{1}+\alpha_{\min }$ \\
\hline
\end{tabular}

TWO-MATERIAL THERMAL PROPERTY INTERPOLATION FORMULAE
Energy conservation

$$
\rho_{\gamma} C_{p \gamma}(u . \nabla T)=\nabla \cdot\left(k_{\gamma} \nabla T\right)
$$

Heat flux Boundary condition: $(k \nabla T) \cdot n=Q$

Solution of these equations requires properties $\mathrm{k}_{\gamma}, \mathrm{C}_{\mathrm{p} \gamma}$ and $\rho_{\gamma}$ which are material dependent. The thermophysical material property at any point on the design domain depends on the sign of level set function and it is defined in Table II. The symbol ' $\mathrm{H}$ ' in the definition represents Heaviside or Unit step function, which takes unit value when LSF is positive and zero value when LSF is negative. To ensure continuity of material properties, a smoothed Heaviside function is used in this formulation given by equation 5 . The derivative of Heaviside function is Delta function and its expression is given in equation 6.

$$
\begin{gathered}
H(\psi)=\frac{1}{2}+\frac{15}{16}\left(\frac{\psi}{h}\right)-\frac{5}{8}\left(\frac{\psi}{h}\right)^{3}+\frac{3}{16}\left(\frac{\psi}{h}\right)^{5} \\
\delta(\psi)=\frac{15}{16 h}\left(1-\left(\frac{\psi}{h}\right)^{2}\right)^{2}
\end{gathered}
$$

The optimization approach considers a temporal evolution of the LSFs based on solution of one Hamilton-Jacobi equation for each LSF, as given in equations 7 and 8.

$$
\begin{aligned}
& \frac{\partial \psi_{1}}{\partial t}=V_{n 1}\left|\nabla \psi_{1}\right| \\
& \frac{\partial \psi_{2}}{\partial t}=V_{n 2}\left|\nabla \psi_{2}\right|
\end{aligned}
$$

If ' $F$ ' is the objective function, which is minimized through topology optimization, then the change in objective function to the change in shape of the material domain is defined as shape sensitivity. The velocity of propagation of level-set function $\left(V_{n}\right)$ is a function of shape sensitivity and it is calculated using the Augmented Lagrangian method [37]. The augmented Lagrangian of this problem is given by:

$$
\begin{aligned}
L=F(\Omega)+ & \lambda_{1}\left(\int_{\Omega} H\left(\psi_{1}\right) d \Omega-V_{1} * V_{\Omega}\right)+ \\
& \lambda_{2}\left(\int_{\Omega} H\left(\psi_{1}\right) H\left(\psi_{2}\right) d \Omega-V_{2} * V_{\Omega}\right)
\end{aligned}
$$

In the above equation $\lambda_{1}$, and $\lambda_{2}$ are Lagrangian multipliers. The second and third terms on the right hand side of this equation denotes the volume constraint on the total solid usage and the second solid usage respectively. Imposition of volume constraint makes the problem a constrained optimization problem (which is well posed) and further, the mass of the solid used influence the cost of the heat sink significantly. So imposing volume constraints helps to restrain the cost, 
indirectly. The Hamilton-Jacobi (HJ) equations are solved using an explicit first order upwind scheme. The time step chosen for marching satisfies the Courant-Friedrichs-Lewy (CFL) [38] condition for stability. Every time the physical problem is solved, the $\mathrm{HJ}$ equations are marched in time in order to obtain the new shape and new level set functions. The velocity of propagation of the level-set functions is obtained by differentiating the Lagrangian with respect to corresponding level-set functions. A volume penalty term is added to ' $\mathrm{V}_{\mathrm{n}}$ ' to ensure volume constraint satisfaction.

$$
\begin{aligned}
& V_{n 1}=F_{1}^{\prime}(\Omega)+\left(\lambda_{1}+\lambda_{2} H\left(\psi_{2}\right)\right) \delta_{1} \\
&+\Lambda_{1}\left(\int_{\Omega} H\left(\psi_{1}\right) d \Omega-V_{1} * V_{\Omega}\right) \\
& V_{n 2}=F_{2}^{\prime}(\Omega)+ \lambda_{2} H\left(\psi_{1}\right) \delta_{2}+ \\
& \Lambda_{2}\left(\int_{\Omega} H\left(\psi_{1}\right) H\left(\psi_{2}\right) d \Omega-V_{2} * V_{\Omega}\right)
\end{aligned}
$$

In the above equations, $F_{1}{ }^{\prime}(\Omega), F_{2}{ }^{\prime}(\Omega)$ are shape sensitivities, and $\Lambda_{1}, \Lambda_{2}$ are volume penalty factors corresponding to $\Psi_{1}$ and $\Psi_{2}$ respectively. $V_{1}, V_{2}$ are volume constraints of total solid and solid2 alone respectively, and $\mathrm{V}_{\Omega}$ is the design domain volume. The optimization procedure seeks to minimize the objective given in equation 12 , subject to thermo-fluid behavior defined by equations 1 to 4 , by the Heaviside constraint given in equation 13 and by volume constraints which define the proportion of the domain that is occupied by each of the constituent materials.

Objective (Thermal Compliance),

$$
\begin{gathered}
F=\int_{\Omega} k \gamma *(\nabla T)^{2} d \Omega \\
H\left(\Psi_{l}\right) u=0
\end{gathered}
$$

Equation 13, constrains the fluid velocity in solid region as zero. The shape sensitivities are obtained by differentiating the objective function with respect to each of the level-set functions. Note that since the flow Reynolds number is of comparable order to the Stokes flow, the self-adjoint nature of Stokes flow and heat conduction equations are exploited and the contribution of Navier-Stokes and Energy equation to shape sensitivity is ignored.

$$
\begin{aligned}
& \left.F_{1}^{\prime}(\Omega)=\left(H_{2} * k s_{2}+\left(1-H_{2}\right) * k s_{1}-k_{f}\right) * \delta_{1} *(\nabla T)^{2}\right) \\
& \left.F_{2}^{\prime}(\Omega)=\left(k s_{2}-k s_{1}\right) * H_{1} * \delta_{2} *(\nabla T)^{2}\right)
\end{aligned}
$$

Dirac-delta functions $\delta_{1}$ and $\delta_{2}$ are derivatives of Heaviside functions $\mathrm{H}_{1}$ and $\mathrm{H}_{2}$ as given in equation 6. A two dimensional optimization study using this formulation is presented by Santhanakrishnan et al. [39].

\section{THREE-MATERIAL LEVEL SET TOPOLOGY OPTIMIZATION MODEL}

For optimization of problems involving three solids and one

\begin{tabular}{|c|c|c|}
\hline Property & Notation & Expression \\
\hline $\begin{array}{l}\text { Thermal } \\
\text { conductivity }\end{array}$ & $\mathrm{K} \gamma$ & $\begin{array}{l}\mathrm{H}_{1} *\left(\mathrm{H}_{2} *\left(\left(1-\mathrm{H}_{3}\right) * \mathrm{ks}_{2}+\mathrm{H}_{3} * \mathrm{ks}_{3}\right)+(1-\right. \\
\left.\left.\mathrm{H}_{2}\right) * \mathrm{ks}_{1}\right)+\mathrm{k}_{\mathrm{f}} *\left(1-\mathrm{H}_{1}\right)\end{array}$ \\
\hline $\begin{array}{l}\text { Specific heat } \\
\text { capacity }\end{array}$ & $\mathrm{Cp} \gamma$ & $\begin{array}{l}\mathrm{H}_{1} *\left(\mathrm{H}_{2} *\left(\left(1-\mathrm{H}_{3}\right) * \mathrm{Cp}_{2}+\mathrm{H}_{3} * \mathrm{Cp}_{3}\right)+\left(1-\mathrm{H}_{2}\right)^{*}\right. \\
\left.\mathrm{Cp}_{1}\right)+\mathrm{C} \mathrm{p}_{\mathrm{f}} *\left(1-\mathrm{H}_{1}\right)\end{array}$ \\
\hline Density & $\rho \gamma$ & $\begin{array}{l}\mathrm{H}_{1} *\left(\mathrm{H}_{2} *\left(\left(1-\mathrm{H}_{3}\right) * \rho_{2}+\mathrm{H}_{3} * \rho_{3}\right)+\left(1-\mathrm{H}_{2}\right) * \rho_{1}\right)+ \\
\rho_{\mathrm{f}} *\left(1-\mathrm{H}_{1}\right)\end{array}$ \\
\hline $\begin{array}{l}\text { Impermeability } \\
\text { factor }\end{array}$ & $\alpha$ & $\left(\alpha_{\max }-\alpha_{\min }\right)^{*} \mathrm{H}_{1}+\alpha_{\min }$ \\
\hline
\end{tabular}
fluid the framework defined in the previous section is extended to consider three LSFs. The correlation between LSF values and material distribution is illustrated in Figure 2. The correlation between the Heaviside function and the material property values are defined in Table III.

TABLE III

THREE-MATERIAL THERMAL PROPERTY INTERPOLATION FORMULAE

The three-material Augmented Lagrangian of this problem is given by:

$$
\begin{gathered}
L=F(\Omega)+\lambda_{1}\left(\int_{\Omega} H\left(\psi_{1}\right) d \Omega-V_{1} * V_{\Omega}\right)+ \\
\lambda_{2}\left(\int_{\Omega} H\left(\psi_{1}\right) H\left(\psi_{2}\right)\left(1-H\left(\psi_{3}\right)\right) d \Omega-V_{2} *\right. \\
\left.V_{\Omega}\right)+\quad \lambda_{3}\left(\int_{\Omega} H\left(\psi_{1}\right) H\left(\psi_{2}\right) H\left(\psi_{3}\right) d \Omega-V_{3} * V_{\Omega}\right)
\end{gathered}
$$

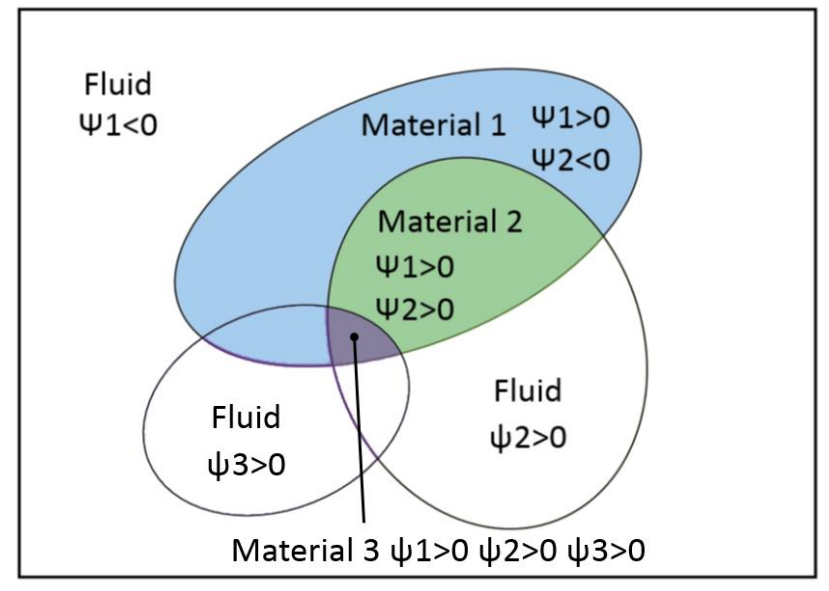

Fig. 2. Design domain and level set function definitions for 3 material case

The level-set convection velocities and shape sensitivities are therefore calculated from the functions defined in Table III.

$$
\begin{aligned}
V_{n 1}=F_{1}^{\prime}(\Omega)+ & \left(\lambda_{1}+\lambda_{2} H\left(\psi_{2}\right)\left(1-H\left(\psi_{3}\right)\right)\right. \\
+ & \left.\lambda_{3} H\left(\psi_{2}\right) H\left(\psi_{3}\right)\right) \delta_{1} \\
+ & \Lambda_{1}\left(\int_{\Omega} H\left(\psi_{1}\right) d \Omega-V_{1} * V_{\Omega}\right) \\
V_{n 2}=F_{2}^{\prime}(\Omega)+ & \left(\lambda_{2} H\left(\psi_{1}\right)\left(1-H\left(\psi_{3}\right)\right)\right. \\
& \left.+\lambda_{3} H\left(\psi_{1}\right) H\left(\psi_{3}\right)\right) \delta_{2} \\
& +\Lambda_{2}\left(\int_{\Omega} H\left(\psi_{2}\right) d \Omega-V_{2} * V_{\Omega}\right)
\end{aligned}
$$




$$
\begin{aligned}
& V_{n 3}=F_{3}^{\prime}(\Omega)-\left(\lambda_{2} H\left(\psi_{1}\right) H\left(\psi_{2}\right)\right. \\
& \left.-\lambda_{3} H\left(\psi_{1}\right) H\left(\psi_{2}\right)\right) \delta_{3} \\
& \quad+\Lambda_{3}\left(\int_{\Omega} H\left(\psi_{3}\right) d \Omega-V_{3} * V_{\Omega}\right) \\
& \left.F_{1}^{\prime}(\Omega)=H_{2} *\left(H_{3} * k s_{3}+\left(1-H_{3}\right) * k s_{2}\right)+\left(1-H_{2}\right) * k s_{1}-k_{f}\right) * \\
& \delta_{1}^{*}(\nabla T)^{2} \\
& \left.F_{2}^{\prime}(\Omega)=H_{1} *\left(H_{3} * k s_{3}+\left(1-H_{3}\right) * k s_{2}\right)-k s_{1}\right) * \delta_{2} *(\nabla T)^{2} \\
& F_{3}^{\prime}(\Omega)=H_{1} * H_{2} *\left(k s_{3}-k s_{2}\right) * \delta_{3} *(\nabla T)^{2}
\end{aligned}
$$

where: $\mathrm{V}_{1}, \mathrm{~V}_{2}$ and $\mathrm{V}_{3}$ are volume constraints of total solid, solid2 alone and solid 3 alone respectively. The Lagrangian multiplier and the volume penalty factor of each of the LSF are updated as follows.

$$
\begin{gathered}
\lambda_{k}=\lambda_{k-1}-\Lambda_{k-1}(\Delta V) \\
\Lambda_{k}=\frac{1}{\beta} \Lambda_{k-1}
\end{gathered}
$$

where $\Delta \mathrm{V}$ is the difference between current material volume to required material volume and $\beta$ is the factor used to update the volume penalty factor.

The initial value of the Lagrangian multipliers, and area penalty factors are chosen appropriately. Each of the level-set functions is re-initialized at regular intervals by time marching the corresponding Eikonal [36] equation given in equations (25) and (26).

$$
\begin{gathered}
\frac{\partial \psi}{\partial t}+w . \nabla \psi=S(\psi o) \\
w=S(\psi o) \frac{\nabla \psi}{|\nabla \psi|}
\end{gathered}
$$

\section{Computational Details}

The topological optimization framework has been applied to the design of multi-material heatsinks in a simplified electronics packaging scenario. Typically, a heatsink would be placed over a high-power active semiconductor device mounted on a printed circuit board (PCB). This has been simplified by considering the PCB and active package as a two-dimensional surface with a steady heat flux through section of this surface. The topological optimization framework is tasked with defining a heatsink in a cuboidal region above a PCB. The computational domain used for this study is illustrated in Figure 3. It is

$$
\text { TABLE IV }
$$

GEOMETRIC PARAMETERS

\begin{tabular}{llll}
\hline \hline Parameter & Symbol & Value & Unit \\
& & & \\
\hline Thermophysical domain length & L1 & 0.7 & M \\
Thermophysical domain width & W1 & 0.7 & M \\
Thermophysical domain height & H1 & 0.3 & M \\
Design domain length & L2 & 0.1 & M \\
Design domain width & W2 & 0.1 & M \\
Design domain height & H2 & 0.1 & M \\
Heat flux length & L3 & 0.01 & M \\
Heat flux width & W3 & 0.01 & $\mathrm{M}$ \\
Heat flux & Q & 20000 & $\mathrm{~J} \cdot \mathrm{M}^{-2} \cdot \mathrm{s}^{-1}$
\end{tabular}

considered to be one quadrant of the total domain, making use of symmetry boundary condition on the two sides to reduce computational costs. Whilst the results obtained from the analyses appear to be symmetrical there may be cases including natural convection by air, in which symmetry is not a valid assumption. Since this study deals only with forced convection cooling, adoption of a symmetry boundary condition is considered to be valid. As previously described, thermofluidic analysis is performed over the entire computational domain in which the topological optimization is confined to a smaller design domain. The geometric parameters used in this study are defined in Table IV. Material properties used in this study are given in Table V. Though in electronic cooling applications air is commonly used fluid, here in this study a methanol/water mixture is used, mainly because of computational reasons. High viscous fluids take less computational time to converge than low viscous air like fluids. It should be noted that the variation in thermal properties of working fluid with respect to temperature is not considered in this study.

The fluid enters the domain through the upper surface at a temperature of $293 \mathrm{~K}$ and velocity corresponding to a Reynolds number of 8 at which the Prandtl number corresponds to 10.5. The fluid exits through two outlet surfaces which have pressure defined as being equal to ambient. The reasoning behind the adoption of a relatively low Reynolds number stems from the non-linear relationship between Reynolds number and computational expense. Convergence of the fluid flow solution, particularly in the presence of porous solid regions within the design domain worsens rapidly as Reynolds number increase, resulting in a significant increase in computational cost. Likewise, the selection of mesh density is guided by computational expense limitations. This study is primarily intended to demonstrate a methodology rather than to assess a

\begin{tabular}{|c|c|c|c|c|}
\hline Material & Property & Symbol & Value & Unit \\
\hline \multirow{3}{*}{ Copper } & Specific heat & $\mathrm{C}_{\mathrm{ps} 1}$ & 385 & $\mathrm{~J} \mathrm{~kg}^{-1} \mathrm{~K}^{-1}$ \\
\hline & Density & $\rho_{\mathrm{s} 1}$ & 8920 & $\mathrm{Kg} \mathrm{m}^{-3}$ \\
\hline & $\begin{array}{l}\text { Thermal } \\
\text { conductivity }\end{array}$ & $\kappa_{\mathrm{s} 1}$ & 400 & $\mathrm{~W} \cdot \mathrm{M}^{-1} \cdot \mathrm{K}^{-1}$ \\
\hline \multirow{3}{*}{ Aluminum } & Specific heat & $\mathrm{C}_{\mathrm{ps} 2}$ & 920 & $\mathrm{~J} \mathrm{~kg}^{-1} \mathrm{~K}^{-1}$ \\
\hline & Density & $\rho_{\mathrm{s} 2}$ & 2700 & $\mathrm{Kg} \mathrm{m}^{-3}$ \\
\hline & $\begin{array}{l}\text { Thermal } \\
\text { conductivity }\end{array}$ & $\kappa_{\mathrm{s} 2}$ & 200 & $\mathrm{~W} \cdot \mathrm{M}^{-1} \cdot \mathrm{K}^{-1}$ \\
\hline \multirow{3}{*}{$\begin{array}{l}\text { Methanol/W } \\
\text { ater mixture }\end{array}$} & Specific heat & $\mathrm{C}_{\mathrm{pf}}$ & 4184 & $\mathrm{~J} \mathrm{~kg}^{-1} \mathrm{~K}^{-1}$ \\
\hline & Density & $\rho_{\mathrm{f}}$ & 1000 & $\mathrm{Kg} \mathrm{m}^{-3}$ \\
\hline & $\begin{array}{l}\text { Thermal } \\
\text { conductivity }\end{array}$ & $\kappa_{\mathrm{f}}$ & 0.4 & $\mathrm{~W} \cdot \mathrm{M}^{-1} \cdot \mathrm{K}^{-1}$ \\
\hline
\end{tabular}
specific problem. As such, we would expect variation in the optimized design with increases in Reynolds number but the timescales of such analyses would rapidly increase beyond the 140 hour 10-core parallel analyses typical of the presented study.

As such, the design domain is discretized with $43 \times 43 \times 43$ hexahedral cells giving a total mesh size of 208,376 elements. Initial level sets are spherical in shape in a manner determined through a parametric study. A total of three different analyses

TABLE V

MATERIAL PROPERTIES 
were performed. The first was a single material baseline, with only copper present. Two copper-aluminum studies were performed, each initialized differently, and yielding substantially different results indicating that design domain has many optimums and the final shape obtained depends on the initialization. In the copper-only analysis the volume constraint was set to 0.25 meaning that the algorithm could distribute 250 cubic centimeters of copper within the 1000 cubic centimeter design domain. The volume constraints for each of the two further cases were 100 cubic centimeters of copper and 150 cubic centimeters of aluminum. These volume constraint values were selected as they were considered indicative of values prevalent in conventional heatsink geometries.

Checkerboards are alternating solid and void regions formed during topology optimization and are mainly reported in studies using the density method. The results of the present level-set method are free from checkerboard issues, as the design variables (level-set function) are solved separately using finitedifference method in Matlab and thermo-fluid equations are solved in Comsol using higher order finite-elements.

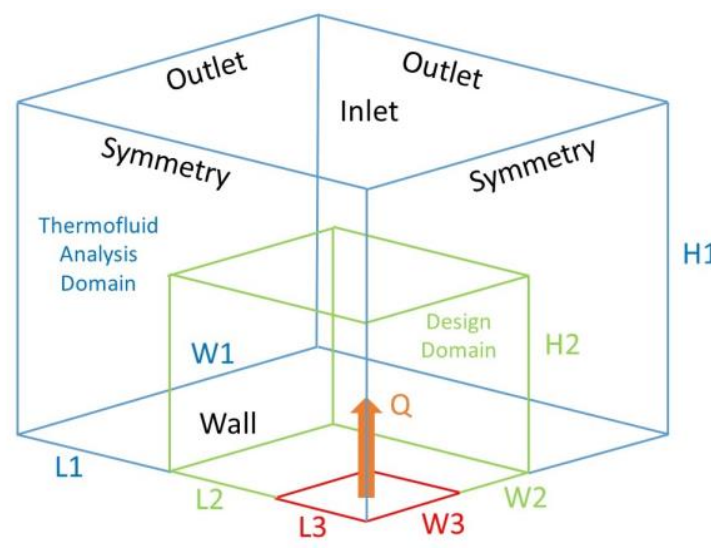

Fig. 3. Illustration of the computational domain

\section{RESULTS \& DiSCUSSION}

\section{A. Multi-material Heat sink design Study}

The results of the three optimization studies are presented in Figures 5 to 7 . Thermal compliance results are presented in Table VI. Each of the simulations is progressed to a fully converged state. Convergence of the Lagrange multiplier and thermal compliance are presented in Figure 4. Each of these analyses require in the order of 80 optimization iterations to reach convergence with a total run time of approximately 140 hours on a 10 Xeon core Workstation. Progression to greater Reynolds numbers results in a worsening in convergence behavior and an increase in computational expense.

Figures 5(a) and 5(b) show the solution obtained for the pure copper baseline scenario. The result obtained from the topological optimization framework is clearly different from a traditional heatsink design. The copper material is predominantly located directly above the heat flux area with a number of branch-like structures protruding toward and through an upper cap region shown in Figure 5(b). The structure is certainly not concentric and has some floating sections. These floating regions result from the lack of a continuity constraint in the optimization process. The algorithm attempts to find the optimal arrangement of material within the design space but is not limited to forming a contiguous structure.
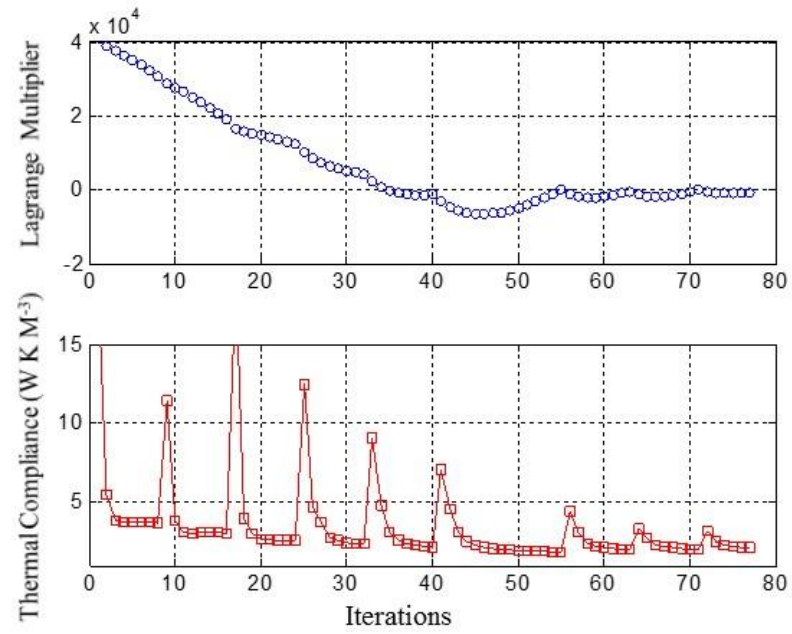

Fig. 4. Optimization convergence metrics
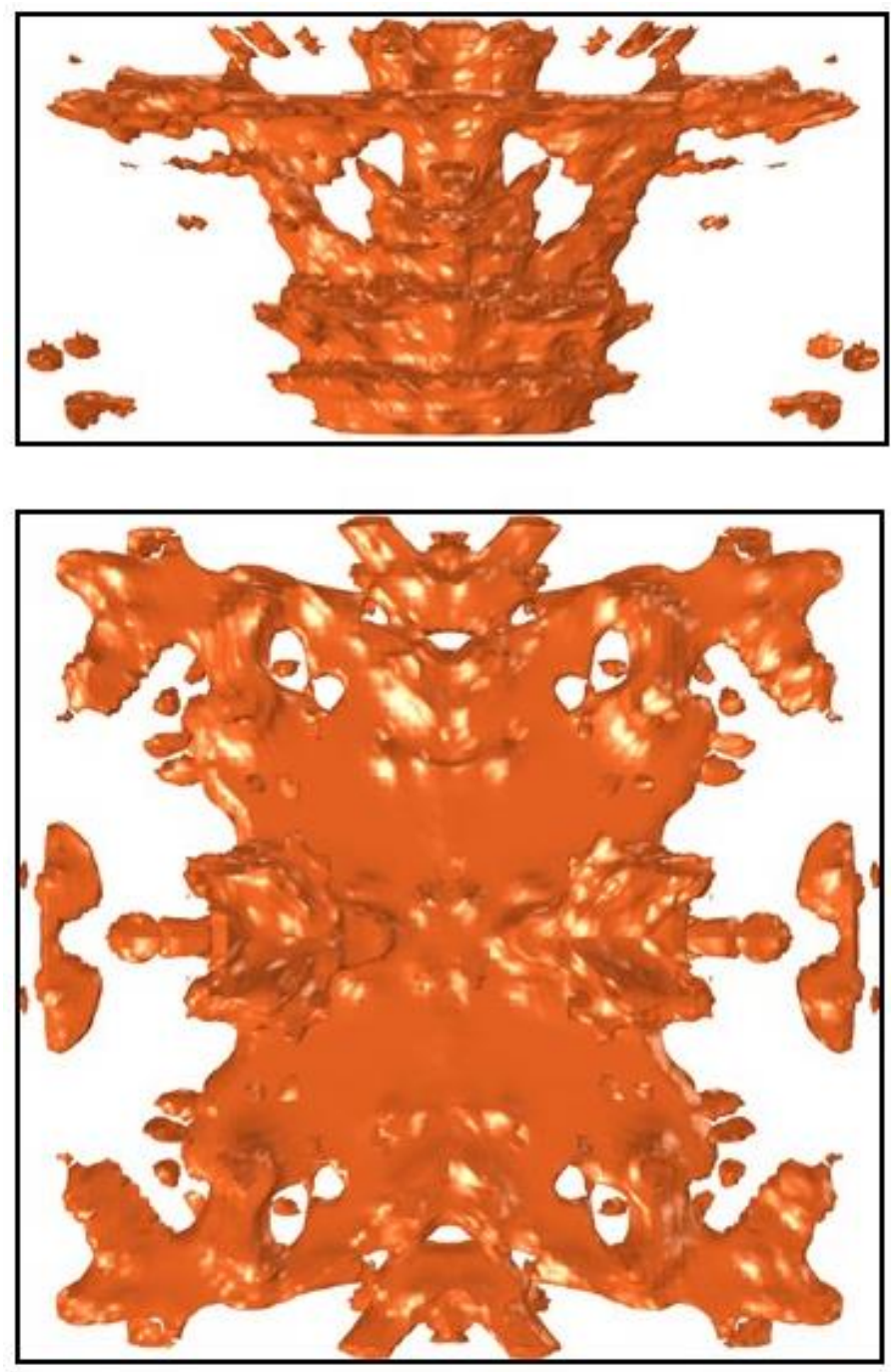

Fig. 5a. Pure copper heatsink (Volume constraint=0.25): top and side views 


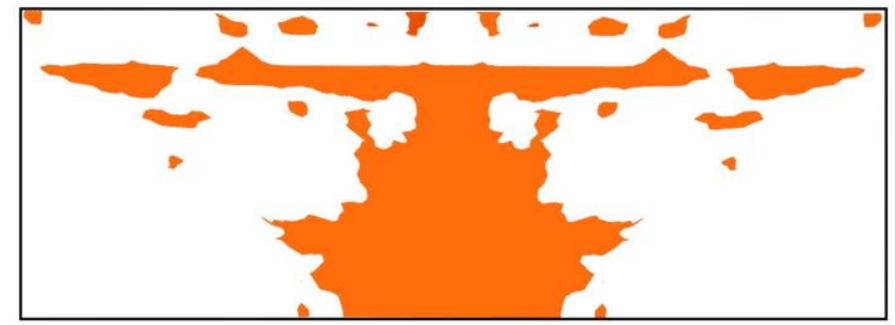

Fig. 5b. Pure copper heatsink (Volume constraint=0.25): cross sectional view
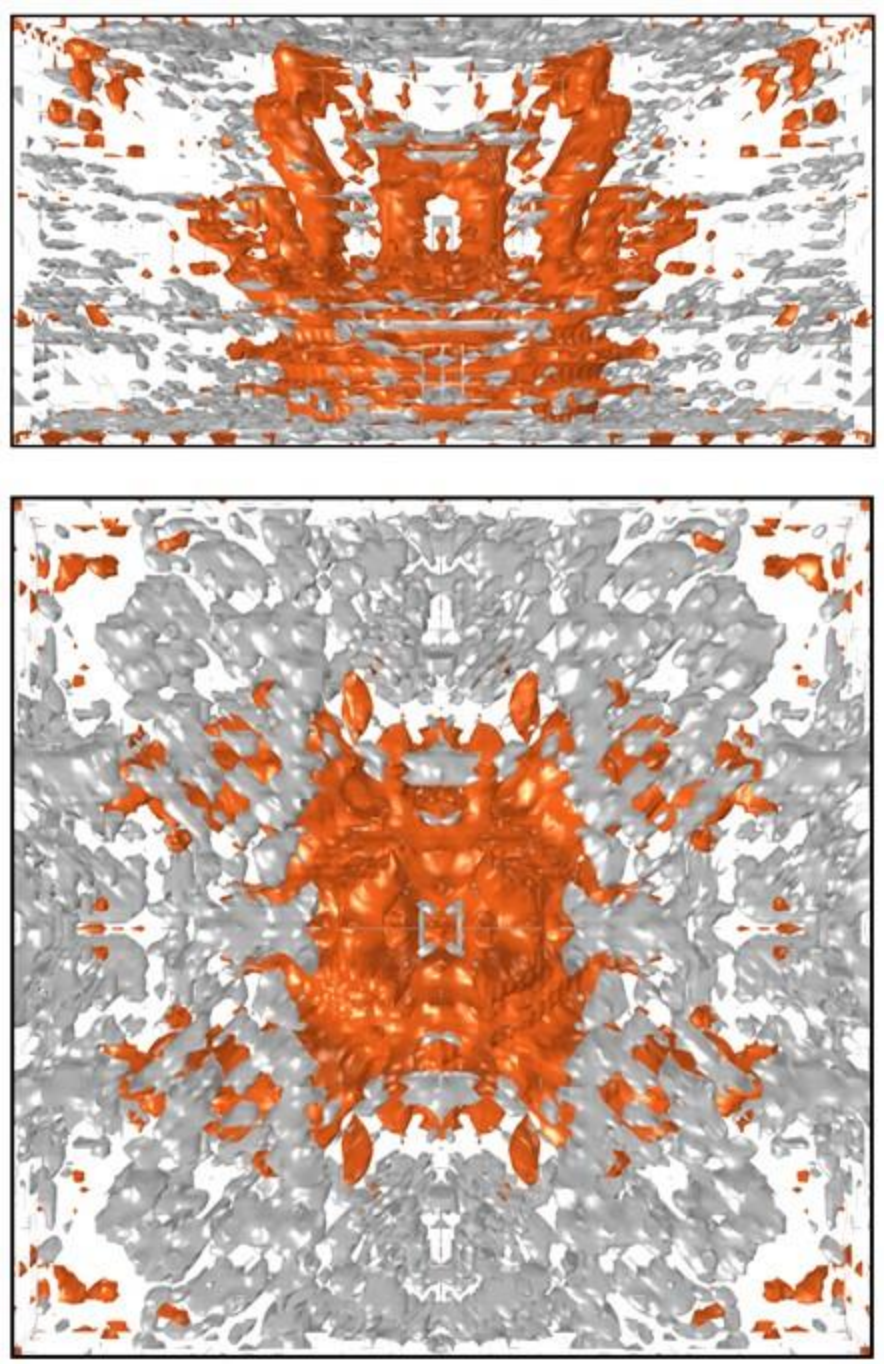

Fig. 6a. Copper-Aluminum heatsink 1: top and side views

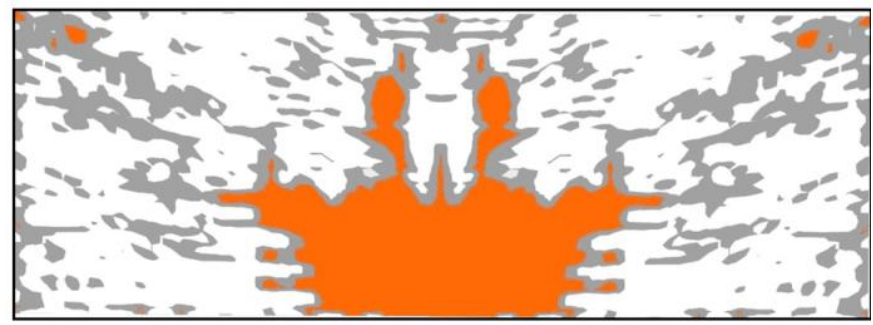

Fig. 6b. Copper-Aluminum heatsink 1: cross sectional view. (Volume constraint of Copper and Aluminum are $0.10 \& 0.15$ respectively)
A mathematical approach to avoid the floating sections would be to augment the optimization algorithm with additional constraints to preclude formation of floating structures. Approaches for countering this mathematically are discussed later. Figures 6(a) and 6(b) show the solution obtained for the first copper-aluminum scenario. In this design, the copper material is again predominantly located directly above the heat flux region and again forms branch like structures.

The aluminum material is predominantly distributed in a series of unconnected or feebly connected regions between the central copper core and the boundaries of the design domain. This is effectively an artifact of the presence of grey cells (half solid and half fluid cells) despite the attempts to counter this through re-initialization. This can be combatted by refining the mesh size or by a number of alternative approaches which are discussed in the later part of this section.

An alternate level set initialization of the copper-aluminum analysis results in a material distribution as depicted in Figure $7 \mathrm{a}$ and $7 \mathrm{~b}$. In this design, aluminum is placed over the heat flux surface while the copper is distributed toward the extremities of the design domain. This analysis has been included to show the sensitivity of this method to level-set initializations. As with all gradient-based optimization approaches, the Level-set topological optimization algorithm is likely to settle in the first encountered minima if there are multiple minima present in the problem domain. Hence, a sequence of studies with differing initializations is required to determine a global optima.

TABLE VI

THERMAL PERFORMANCE RESULTS

\begin{tabular}{lcl}
\hline \hline Case & $\begin{array}{c}\text { Thermal Compliance } \\
\left(\mathrm{W} \mathrm{K} \mathrm{M} \mathrm{M}^{-3}\right)\end{array}$ & $\begin{array}{l}\text { Maximum } \\
\text { Temperature }(\mathrm{K})\end{array}$ \\
\hline Pure copper & 2.30 & 316.5 \\
Copper-Aluminum 1 & 2.16 & 316.5 \\
Copper-Aluminum 2 & 3.25 & 317.1
\end{tabular}

The overall thermal compliance results for the three designs are provided in Table VI. From these results it can be seen that the performance of the first copper-aluminum design performs marginally better than the pure copper design while the second copper-aluminum design, with aluminum located centrally, performs poorly. The superior performance of the copperaluminum design over the pure copper design is clearly counterintuitive. An electronics engineer would expect the higher thermal conductivity of the copper material to provide better performance. This discrepancy can be considered to arise through the pure-copper design resulting from an optimal design differing from the global optima, with the copperaluminum design finding either a global optimum or superior local optimum. Additionally, the increase in discrete floating sections apparent in the copper-aluminum design may have an influence through evening out temperature gradients present within the design domain. 

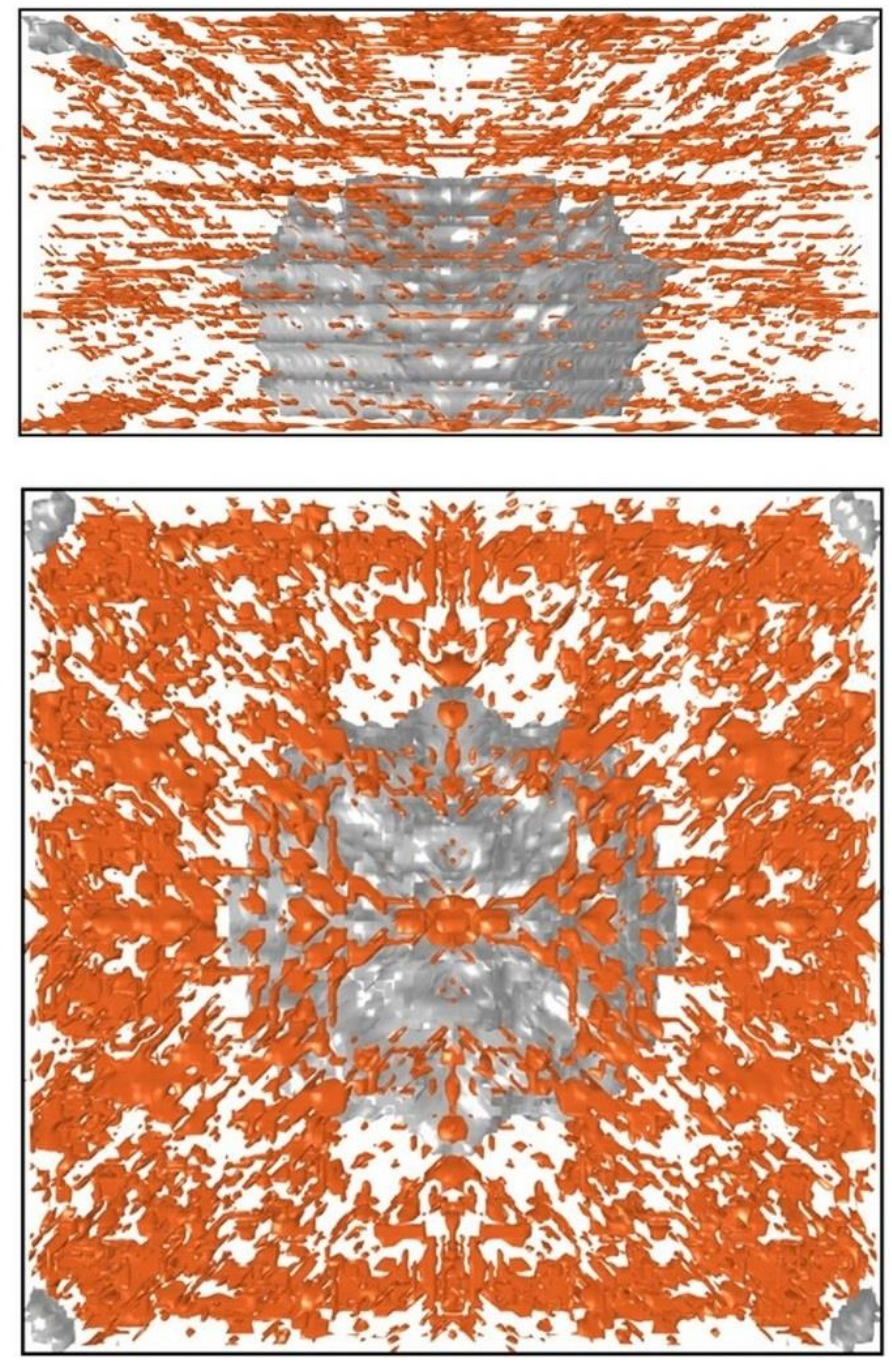

Fig. 7(a). Copper-Aluminum heatsink 2: top and side views

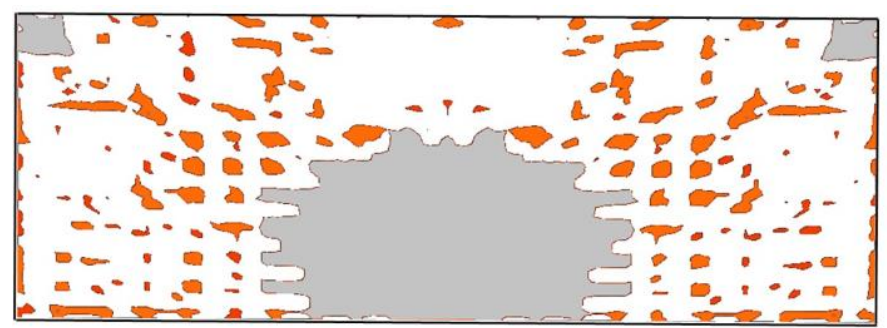

Fig. 7b. Copper-Aluminum heatsink 2: cross sectional view (Volume constraint of Copper and Aluminum are $0.10 \& 0.15$ respectively)

This study is intended to demonstrate the applicability of topological optimization to the design of multi-material heatsinks. The results presented demonstrate that this is the case. However, few issues are clearly present. The future development of topological optimization algorithms must address these issues. The designs presented feature disconnected floating bodies, which are mathematically beneficial to minimize the objective, but would be unfeasible to manufacture. These floating objects could be avoided through a simple change such as optimizing for a relaxed objective value or through re-defining the objective function. Alternatively, regularization techniques such as perimeter filtering [40],
Tikhonov regularization [41] or sensitivity filtering [42] could be integrated into the algorithm. Alternatively, or additionally, thin feature control can be implemented to prevent the formation of thin structures. Chen [43] employed a quadratic energy functional in the objective function of the topology optimization, to introduce interactions between different points on the structural boundary to favor strip-like shapes with specified widths. Allaire et al. [44] compared different thickness control methods and recommended an energy functional based thickness control methods to overcome this issue.

The nature of gradient based optimization approaches results in analyses commonly finding the nearest optimum (as dictated by the sensitivity) that could be local or global. This is typically tackled through performing a series of studies with differing initializations. This clearly increases the, already substantial, computation cost of such studies. The ability to perform such a study and the limits on design domain mesh size are limited by available computational resources. The accuracy in modelling the muli-material LSM is constrained by this, with marginal improvement possible with adoption of a finer mesh. Compared to single material TO, multi-material $\mathrm{TO}$ require finer mesh and frequent re-initialization as each material is represented as a product of two Heaviside functions (Table II). If Heaviside function values are less than 1 , then their product will be much less than 1 , hence leading to more grey areas. As such, there are some issues relating to the robustness of the solution obtained regard to the ability to obtain global, rather than local, optima, sensitivity of the design to small changes in model definition. These matters could be addressed through use of a large parallel HPC system, assessing optimal designs for a wide range of differing initialization patterns and through refinement of domain discretization to evaluate variation in optimal design with analysis resolution.

This study is carried out using Ersatz material mapping method and hence the solids created are porous. The drawback of this method is that it is not possible to impose a no-slip condition on the solid walls. This results in pressure diffusion across the solid boundaries and the porosity approach also leads to flow convergence issues. An alternative would be to utilize an XFEM mapping along with level-set method. This would overcome these disadvantages but, again, at an increased computational cost and could additionally be an issue addressed in future work.

\section{B. Benchmarking Study}

In order to benchmark the level-set topology optimization method, the performance of the 3D single material (copper) heat sink is compared against a conventional pin-fin heat sink in a separate conjugate heat transfer CFD study in Comsol. In this 3D CFD study, it is ensured that the computational domain and the properties of the materials are exactly same as the one used in the 3D topology optimization study. So here again, only one quarter of the domain is modelled exploiting symmetry boundary conditions (Figure 3). 
In this validation study the isolated regions which were part of the optimized pure copper heat sink (Figure 5a) are ignored and a Heaviside function threshold value of 0.9 is used to extract the optimized heat sink geometry from Comsol. The resulting geometry has a volume of $1.141 \mathrm{e}-4 \mathrm{~m}^{3}$ hence a pin-fin heat sink is also designed to have exactly the same volume (Figure 8). Each fin of the pin-fin heat sink has a cross section of side $0.00703 \mathrm{~m}$ and a height of $0.0915 \mathrm{~m}$ including the fin base of height $0.0025 \mathrm{~m}$. Fin base size is $0.1 \times 0.1 \mathrm{~m}$ and the inter-fin spacing is kept uniform at $0.015 \mathrm{~m}$.

Tetrahedral elements are used for the CFD simulation of both the pin-fin heat sink and the LSM designed heat sink. The total

TABLE VII

CFD RESULTS

\begin{tabular}{l|c|l}
\hline \multicolumn{3}{c}{ CFD RESULTS } \\
Case & $\begin{array}{c}\text { Thermal Compliance } \\
\left(\mathrm{W} \mathrm{K} \mathrm{M} \mathrm{M}^{-3}\right)\end{array}$ & $\begin{array}{l}\text { Maximum } \\
\text { Temperature }(\mathrm{K})\end{array}$ \\
\hline $\begin{array}{l}\text { Pin-fin Heat sink } \\
\mathrm{Q}=20 \mathrm{~kW} / \mathrm{m}^{2}\end{array}$ & 4.1712 & 295.61 \\
$\mathrm{Q}=40 \mathrm{~kW} / \mathrm{m}^{2}$ & 16.6846 & 298.07 \\
\hline $\begin{array}{l}\text { LSM Heat sink } \\
\mathrm{Q}=20 \mathrm{~kW} / \mathrm{m}^{2} \\
\mathrm{Q}=40 \mathrm{~kW} / \mathrm{m}^{2}\end{array}$ & 4.1117 & 295.10 \\
& 16.4443 & 297.05 \\
\hline
\end{tabular}

number of elements used to discretize the computational domain is 1.25 million in both the cases. It is also ensured that inter-fin spacing of pin-fin heat sink had 10-30 tetrahedral elements to accurately capture the convective heat transfer effect. Simulations are solved using the segregated solver present within the Comsol.
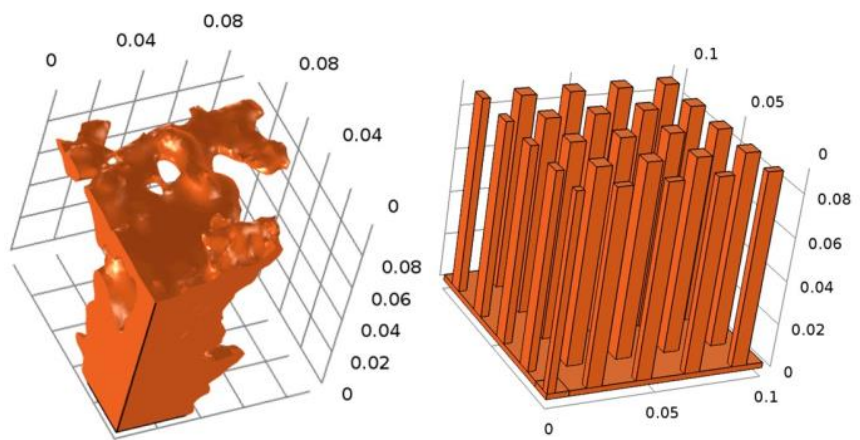

Fig. 8. LSM and Conventional pin-fin heat sinks
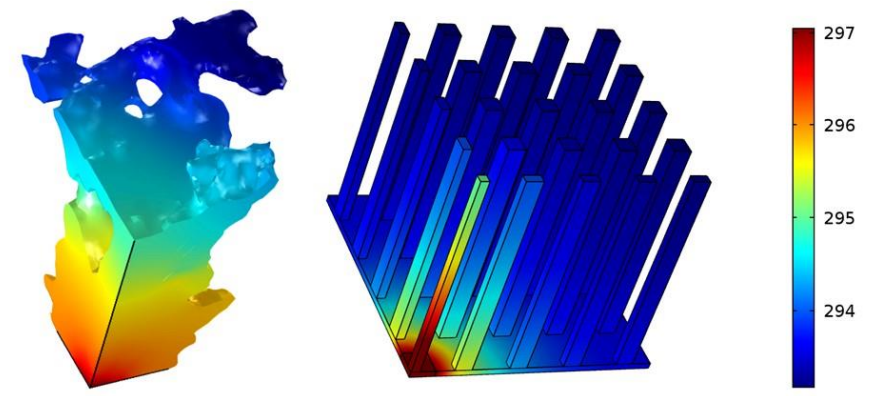

Fig. 9. Temperature (K) contour from the CFD study

The CFD study is conducted for two different heat flux values, $20 \mathrm{~kW} / \mathrm{m}^{2}$ and $40 \mathrm{~kW} / \mathrm{m}^{2}$ as the temperature rise with respect to the ambient temperature $(293 \mathrm{~K})$ is considerably less $\left(2-5^{\circ} \mathrm{C}\right)$. The maximum temperature reported by the CFD study is considerably lower than the value reported during optimization, mainly because the solids created during optimization are porous solids and the thermal coupling between the solid and fluid is not perfectly modelled, whereas in CFD the thermal coupling is perfectly modelled. The CFD results show that the LSM heat sink has slightly lower thermal compliance value and maximum temperature value than the conventional heat sink (Table VII \& Figure 9). This result validates that LSM is capable of designing heat sinks which are on par or slightly better than the conventional heat sinks.

It should also be noted that, the objective of optimization did not directly consider the convective cooling effect but only minimized the thermal compliance of the design domain. Use of such specific objective, might yield much better designs [23] than the present one. It is also worth noting that, the conventional pin-fin heat sink is not optimized for $\mathrm{Re}=8$, so this study should be considered to give only a qualitative idea about the LSM performance.

The validation obtained for single material heat sink can be extended to multi-material heat sink design, but nevertheless, the formulation used in this study has to be improved in terms of preventing the floating structure formation and better reinitialization capabilities. The primary benefit of multi-material LSM is that it is capable of determining the optimal distribution of multiple materials within a set of imposed design constraints. Design of multi material heatsinks using traditional design methods is rather limited. As such, this benchmarking study does omits consideration of multi-material designs.

\section{CONCLUSIONS}

This study sets out to determine the applicability of the levelset topological optimization algorithm to the design of multi material heat sinks within a simplified electronics thermal management scenario. Further, this study is significant as it extends the state-of-the-art to multi-material analysis in situations involving forced convective cooling. The results presented indicate that level-set topological optimization method can provide interesting and competent heat sink shapes taking into account both conduction and convection cooling. The 3D benchmarking study proves that the optimized heat sinks are marginally better than conventional heat sinks. Though the paper is focused on forced convective cooling at $\operatorname{Re}=8$, the method can be extended to natural and mixed convections and also to high Reynolds numbers through proper formulation. The paper also details the limitations and challenges of the presented level-set method and suggests a number of approaches that could be adopted to overcome these as part of a future study.

\section{ACKNOWLEDGEMENTS}

The authors wish to acknowledge the University of Greenwich Vice Chancellor's scholarship scheme for providing the funding for this study. 


\section{REFERENCES}

[1] M.P. Bendsoe and N. Kikuchi, "Generating optimal topologies in structural design using a homogenization method", Comput. Methods Appl. Mech. Eng., Vol. 71, no.2, pp.197-224,1988

[2] M. P. Bendsøe, O. Sigmund, "Topology Optimization: Theory, Methods and Applications", Springer, second edition, 2004.

[3] K. Svanberg, "Method of Moving Asymptotes - a New Method for Structural Optimization", Int. J. Numerical Methods Engg., vol. 24, no. 2, pp. 359-373, Feb. 1987.

[4] M.Y. Wang, X. Wang, and D. Guo, "A level set method for structural topology optimization", Comput. Methods Appl. Mech. Eng., vol.192, pp. 227-246, 2003.

[5] G. Allaire, F. Jouve, and A.M. Toader, "Structural optimization using sensitivity analysis and a level-set method", J. Computational Physics, vol.194, no.1, pp.363-393, 2004

[6] T. Borrvall, J. Petersson, "Topology optimization of fluids in stokes flow", Int. J. Numerical Methods Fluids, Vol. 41, pp. 77-107, 2003.

[7] L.H. Olesen, F. Okkels, H. Bruus, "A high-level programming-language implementation of topology optimization applied to steady-state NavierStokes flow", Int. J. Numerical Methods Engg., Vol. 65, No. 7, pp. 9571001, 2006.

[8] V. Challis, J.K. Guest, "Level set topology optimization of fluids in Stokes flow", Int. J. Numerical Methods Engg., vol.79, no.10, pp.12841308, 2009.

[9] S. Zhou, Q. Li, "A variational level set method for the topology optimization of steady-state Navier-Stokes flow", J. Computational Physics, vol. 227, no. 24, 2008.

[10] S. Kreissl and K. Maute, "Level set based fluid topology optimization using the extended finite element method", Struct. Multidiscip. Optim., vol. 46, no. 3, pp.311-326, 2012.

[11] O. Sigmund and S. Torquato, "Design of materials with extreme thermal expansion using a three-phase topology optimization method", J. Mech. Phys. Solids, vol. 45, no.6, pp.1037-1067, 1997.

[12] M. Wang and X. Wang, "Color level sets: a multi-phase method for structural topology optimization with multiple materials", Comput. Methods Appl. Mech. Eng., vol. 193, no.6, pp.469-496, 2004.

[13] Y. Wang, Z. Luo, Z. Kang and N. Zhang, "A multi-material level setbased topology and shape optimization method", Comput. Methods Appl. Mech. Eng., Vol. 283, pp.1570-1586, 2015.

[14] G. Allaire, C. Dapogny, G. Delgado and G. Michailidis, "Multi-phase Structural Optimization via a Level-Set Method", ESAIM: Control, Optimization and Calculus of Variations, vol. 20, no.2, pp. 576-611, 2014.

[15] G. Michailidis, "Manufacturing Constraints and Multi-phase shape and Topology Optimization via a Level-set method", Optimization and control, Ecole Polytechnique X, 2014.

[16] L. Yin, G. Ananthasuresh, "Topology optimization of compliant mechanisms with multiple materials using a peak function material interpolation scheme", Struct. Multidiscip. Optim., vol.23, no.1, pp.4962, 2001.

[17] T. Gao, W. Zhang and P. Duysinx, "A bi-value coding parameterization scheme for the discrete optimal orientation design of the composite laminate", Int. J. Numerical Methods Engg., vol. 91, no.1, pp.98-114, 2012.

[18] M. Bruyneel, "SFP - a new parameterization based on shape functions for optimal material selection: application to conventional composite plies", Struct. Multidiscip. Optim., vol.43, no.1, pp.17-27, 2011.

[19] R. Tavakoli, S.M. Mohseni, "Alternating active-phase algorithm for multimaterial topology optimization problems: a 115-line MATLAB implementation”, Struct. Multidiscip. Optim., vol. 49, no.4, pp.621-642, 2014.

[20] S. Zhou and M.Y. Wang, "Multimaterial structural topology optimization with a generalized Cahn-Hilliard model of multiphase transition", Struct. Multidiscip. Optim., vol. 33, no.2, pp.89-111, 2006.

[21] E.M. Dede, "Multiphysics topology optimization of heat transfer and fluid flow systems", Proc. COMSOL Conference, Boston, 2009.

[22] G.H. Yoon, "Topological design of heat dissipating structure with forced convective heat transfer". J. Mechanical Science and Technology, vol. 24, no. 6, pp.1225-1233, 2010.

[23] E.M. Dede, S.N. Joshi and F. Zhou, "Topology optimization, additive layer manufacturing, and experimental testing of an air-cooled heat sink", J. of mechanical design, vol.137, 2015.

[24] J. Alexandersen, O. Sigmund and N. Aage, "Large scale three dimensional topology optimisation of heat sinks cooled by natural convection", Int. J. of Heat and Mass Transfer, vol.100, pp.976-891, 2016.

[25] K. Yaji, T. Yamada, S. Kubo and S. Nishiwaki, “A topology optimization method for a coupled thermal-fluid problem using level set boundary expressions", Int. J. of Heat and Mass transfer, vol.81, pp.878-888, 2015.

[26] P. Coffin, and K. Maute, "Level set topology optimization of cooling and heating devices using a simplified convection model", Struct. Multidiscip. Optim., vol. 53, no.5, pp.985-1003, 2016.

[27] A. Bejan, "Constructal-theory network of conducting paths for cooling a heat generating volume," International Journal of Heat and Mass Transfer, vol. 40, p. 799-816, 1996

[28] R. Bornoff and J. Parry, "An additive design heat sink geometry topology identification and optimisation methodology," in SEMI-THERM Conference, San Jose, 2015

[29] R. Bornoff, J. Wilson and J. Parry, "Subractive design: A novel approach to heatsink improvement," in SEMITHERM Conference, San Jose, 2016.

[30] C.J.M. Lasance and A. Poppe, "Thermal management for LED applications", Springer Publications, 2014 (Chapter 9: Heat sink basics for industrial applications).

[31] C. Zhuang, Z. Xiong, and H. Ding, "Topology optimization of multimaterial for the heat conduction problem based on the level set method", Engineering Optimization, vol. 42, no.9, pp.811-831, 2011.

[32] K. Long, X. Wang and X. Gu, "Multi-material topology optimization for the transient heat conduction problem using a sequential quadratic programming algorithm", Engineering optimization, 2018

[33] T. Dbouk, "A review about the engineering design of optimal heat transfer systems using topology optimisation," Applied thermal engineering, vol. 112 , pp. 841-854, 2017

[34] https://www.mathworks.com/products/matlab.html [Last accessed: 13 July 2018]

[35] http://www.comsol.com [last accessed: $13^{\text {th }}$ July, 2108]

[36] J.A. Sethian, "Level set methods and fast marching methods: evolving interfaces in computational geometry, fluid mechanics, computer vision and material science", Cambridge University Press, Cambridge, 1999.

[37] J. Nocedal and S.J. Wright, "Numerical Optimization", Springer series in operation research, Springer-Verlag, 1999.

[38] R. Courant, K. Friedrichs and H. Lewy, "Über die partiellen Differenzengleichungen der mathematischen Physik", Mathematische Annalen (in German), vol. 100, no. 1, pp.32-74, 1928.

[39] M. Santhanakrishnan, T. Tilford, C. Bailey, "Multi-material level set based topology optimization of convectively cooled heat sinks", Proceedings of $6^{\text {th }}$ European Conference on Computational Mechanics, Glasgow, UK, 2018.

[40] R.B. Haber, C.S. Jog and M.P. Bendsoe, "A new approach to variabletopology shape design using a constraint on perimeter", Struct. Multidiscip. Optim., vol. 11, no.1, pp.1-12, 1996.

[41] A.N. Tikhonov, A.V. Goncharsky, V.V. Stepanov and A.G. Yagola, "Numerical methods for the solution of ill-posed problems", Springer, New York, 1995.

[42] O. Sigmund, "Design of material structures using topology optimization", $\mathrm{PhD}$ thesis, Department of Solid Mechanics, Technical University of Denmark, 1994.

[43] S. Chen, M.Y. Wang and A.Q. Liu, "Shape feature control in structural topology optimization", Computer-Aided Design, vol. 40, no.9, pp.951962, 2008.

[44] G. Allaire, F. Jouve and G. Michailidis, "Thickness control in structural optimisation via a level set method," Struct. Multidiscip. Optim., vol. 53, pp. 1349-1382, 2016. 


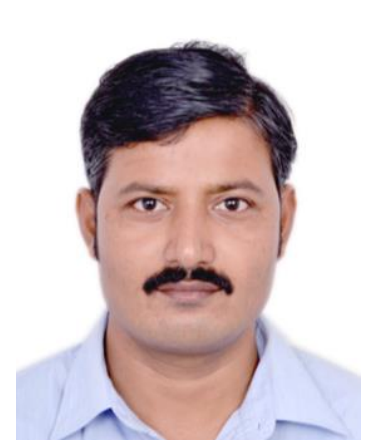

Mani S. Santhanakrishnan received the B.Tech. degree in Aeronautical Engineering from Madras Institute of Technology, Chennai and the M.Eng. degree in Aerospace Engineering from Indian Institute of Science, Bangalore, India in 2000 and 2006 respectively. $\mathrm{He}$ has worked as a Research Engineer, at General Electric, Bangalore from 2007 to 2010 and as a Lead Engineer at Airbus Engineering Centre India from 2010 to 2015. He has completed his $\mathrm{PhD}$ in the University of Greenwich, UK in 2018. He has authored 2 patents, 8 conference papers and a journal article. His research interests are Topology optimization, Heat transfer, Aerodynamics, CFD and Turbomachinery.

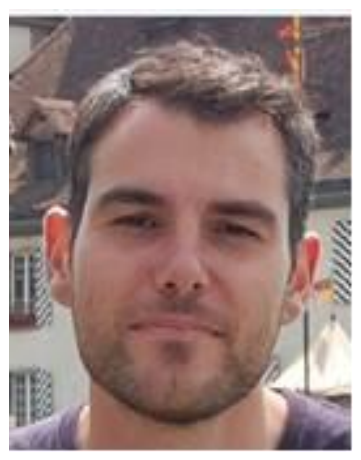

Dr. Tim Tilford obtained a Bachelor's degree in Aeronautical Engineering from Queen Mary College, University of London, United Kingdom, in 1998 and subsequently a Master's Degree in Computational Fluid Dynamics from the University of Greenwich, United Kingdom, in 1999. He obtained a $\mathrm{PhD}$ in Computational Mechanics from the University of Greenwich in 2013. He has been a Research Fellow and subsequently a Senior Lecturer at the University of Greenwich since 2002. His primary research interests are in numerical analysis of multiphysics/multi-scale problems and high performance parallel computing. Dr. Tilford is a Fellow of the Institute of Mathematics and its Applications, a Chartered Mathematician and a Chartered Scientist and an IEEE Member.

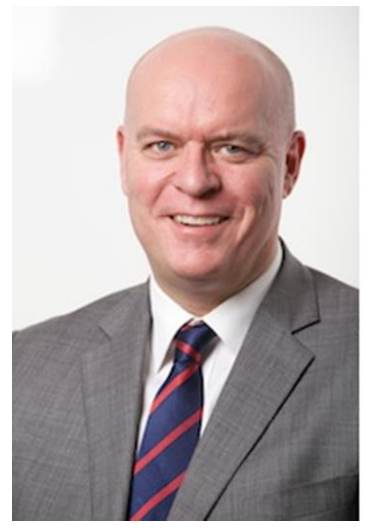

Prof. Chris Bailey (A'00-M'03SM'05) received the MBA degree in technology management from the Open University, Milton Keynes, U.K., in 1996, and the Ph.D. degree in computational modelling from Thames Polytechnic, London, U.K., in 1988. He was a Research Fellow of Materials Engineering with Carnegie Mellon University, Pittsburgh, PA, USA, for three years. He is currently a Professor of the Computational Mechanics and Reliability Group, University of Greenwich. His current research interests include the development of virtual prototyping tools based on multiphysics modelling and numerical optimization. Prof. Bailey is a Committee Member with the International Microelectronics and Packaging Society and the Innovative Electronics Manufacturing Research Centre, U.K. 